\title{
Espessura corneana central e densidade das células endoteliais corneanas centrais após trabeculectomia com e sem mitomicina $\mathrm{C}$
}

\section{Central corneal thickness and central corneal endothelial cells density after trabeculectomy with and without mitomycin C}

Rafael Vidal Mérula', Alberto Diniz Filho', Roberto de Alencar Gomes², Sebastião Cronemberger', Nassim Calixto

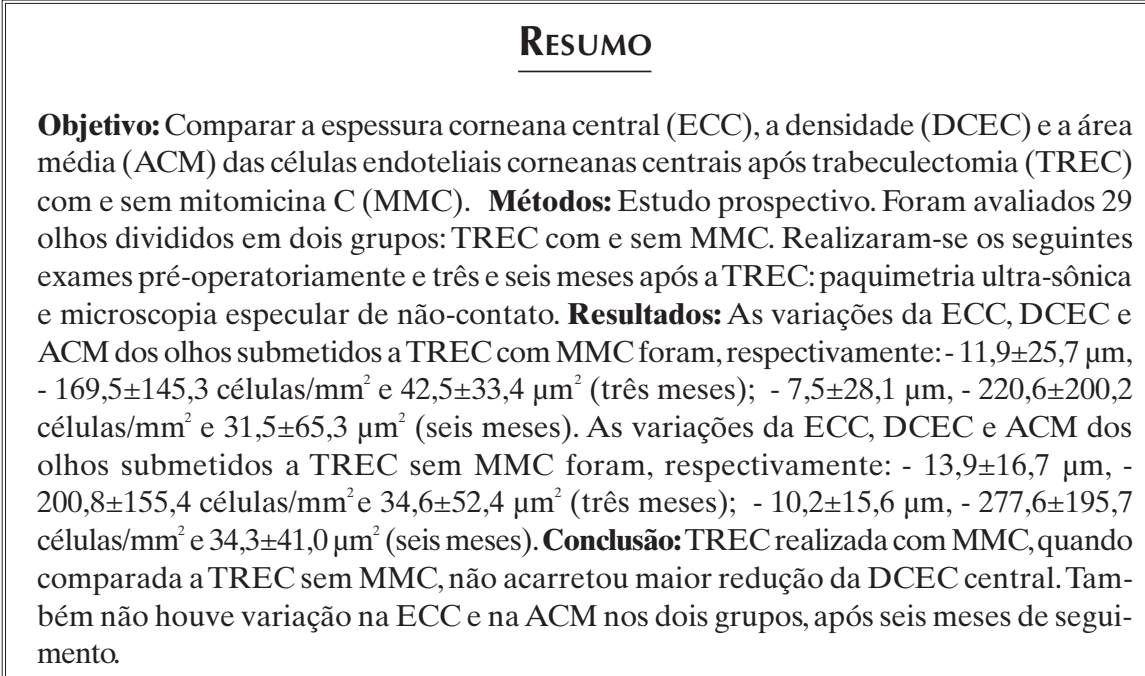

Descritores: Córnea; Endotélio; Contagem de células; Microscopia; Trabeculectomia; Mitomicina

\footnotetext{
${ }^{1}$ Pós-graduando da Faculdade de Medicina da Universidade Federal de Minas Gerais - UFMG - Belo Horizonte (MG), Brasil. Ex-Fellow do Serviço de Glaucoma do Hospital São Geraldo/Hospital das Clínicas da Universidade Federal de Minas Gerais UFMG- Belo Horizonte (MG), Brasil.

${ }^{3}$ Professor Titular da Disciplina de Oftalmologia da Faculdade de Medicina da Universidade Federal de Minas Gerais - UFMG- Belo Horizonte (MG), Brasil;

Trabalho realizado no Serviço de Glaucoma do Hospital São Gerado/Hospital das Clínicas da Universidade Federal de Minas Gerais - UFMG- Belo Horizonte (MG), Brasil.

Recebido para publicação em: 13/11/2007 - Aceito para publicação em 16/2/2008
} 


\section{INTRODUÇÃO}

$\mathbf{O}$ uso de mitomicina $\mathrm{C}(\mathrm{MMC})$ per-operatória com o objetivo de aumentar o sucesso nas trabeculectomias, principalmente em casos de glaucoma de difícil controle, está estabelecido. Entretanto, este importante antimitótico está relacionado com algumas complicações pós-operatórias, como: bolsas fistulantes de paredes finas, hipotonia ocular e descolamento de coróide, além de ser potencialmente tóxico para o segmento anterior, especialmente para as células endoteliais corneanas, ${ }^{(1)}$ bem como, aumenta a incidência de infecções tardias.

É notório que mesmo sem a utilização de MMC há redução da densidade das células endoteliais corneanas (DCEC) após alguns procedimentos cirúrgicos. Uma diminuição de $6 \%$ a $27 \%$ na DCEC já foi demonstrada após extração intracapsular de catarata, extração extracapsular de catarata, facoemulsificação e ceratoplastia penetrante..$^{(2)}$

A redução da DCEC também é observada em pacientes que apresentam crise aguda de glaucoma primário de ângulo fechado e em pacientes com glaucoma crônico de ângulo estreito; entretanto, a população de células endoteliais em olhos de pacientes com crises subagudas de glaucoma de ângulo estreito, e nos olhos contralaterais de pacientes com glaucoma primário de ângulo fechado não é significativamente diferente da média encontrada em pessoas normais. ${ }^{(3)}$ Alguns autores acreditam que pacientes glaucomatosos têm menor DCEC e que esta redução varia com o tipo de glaucoma. ${ }^{(4)}$ Além disso, a diminuição da DCEC está relacionada com o tempo de permanência da pressão intra-ocular ( $\mathrm{Po})$ elevada, sendo aquela maior quanto maior o tempo da persistência da elevação da Po.(5)

Acrescente-se que a diminuição na DCEC é verificada em pacientes portadores de glaucoma que são submetidos a trabeculectomia (TREC), principalmente quando evoluem com câmara anterior rasa no pós-operatório. Observa-se que o toque iridocorneano acarreta uma perda de $10 \%$ das células endoteliais e o toque córneolenticular é ainda mais traumático ao endotélio devendo ser tratado imediatamente. ${ }^{(6.8)}$

Alguns estudos ao avaliarem a espessura corneana central (ECC), concluíram que esta é significativamente maior em pacientes com crise aguda de glaucoma de ângulo fechado, o que não ocorre nos demais subtipos de glaucoma de ângulo fechado. ${ }^{(3)}$ Outros estudos observaram que a ECC é maior em pacientes hipertensos oculares. ${ }^{(5)}$
O presente estudo foi proposto com o intuito de analisar questões críticas relacionadas ao uso da MMC per-operatória nas trabeculectomias: acometimento da DCEC e variações da ECC no período de seis meses de pós-operatório.

\section{Овjetivo}

Avaliar a espessura corneana, a densidade e a área média das células endoteliais na região central da córnea em pacientes submetidos à TREC, através de análise comparativa entre as cirurgias realizadas com e sem a utilização de MMC.

\section{Métodos}

Foi realizado um estudo prospectivo no qual foram selecionados para avaliação de 29 olhos de 27 pacientes acompanhados no Serviço de Glaucoma do Hospital São Geraldo - Hospital das Clínicas/UFMG. O critério de inclusão foi: pacientes terem sido submetidos a apenas uma TREC com ou sem MMC; e os critérios de exclusão foram: trabeculectomia prévia, intervenção cirúrgica durante o acompanhamento pós-operatório (agulhamento, nova cirurgia antiglaucomatosa ou cirurgia de catarata), leucomas ou opacidades corneanas, edema de córnea, toque iridocorneano ou córneolenticular no pós-operatório das trabeculectomias e falha no acompanhamento.

Os 29 olhos foram distribuídos em dois grupos: TREC com MMC e TREC sem MMC.Além disso, foram registrados de todos os pacientes os seguintes dados: idade, olho operado, sexo, relação escavação/disco (E/D), diagnóstico, Po pré-operatória e número de medicações hipotensoras oculares pré-operatórias.

ATREC foi padronizada e realizada pelos "fellows" do Serviço de Glaucoma sob supervisão de preceptoria qualificada: incisão conjuntival nasal superior junto ao fórnice, retalho conjuntival base límbica, retalho escleral retangular (3 $\mathrm{mm} \times 4 \mathrm{~mm}$ ), retirada de retângulo límbico ( $1 \mathrm{~mm} \times 2 \mathrm{~mm})$, iridectomia nasal superior, sutura do retalho escleral com dois pontos de Nylon ${ }^{\circledast} 10-0$, sutura conjuntival contínua com Vicryl ${ }^{\circledR}$ 7-0. Também padronizou-se o emprego da MMC per-operatória: utilizou-se pequeno pedaço de algodão embebido com MMC na concentração de $0,25 \mathrm{mg} / \mathrm{ml}$, deixando-o no espaço subtenoniano durante 5 minutos, evitando contato com as bordas da incisão conjuntival; após a retirada do algodão lavou-se o leito escleral e o fundo de saco conjuntival superior com $40 \mathrm{ml}$ de soro fisiológico a $0,9 \%$. 
Realizaram-se os seguintes exames na região central da córnea: paquimetria ultra-sônica (DGH 5100e) e microscopia especular de não-contato (Konan SP 8000). Pela microscopia especular foram avaliadas: a área celular média e a DCEC centrais. A paquimetria ultra-sônica e a microscopia especular foram realizadas pré-operatoriamente e três e seis meses após a TREC. As medidas foram feitas três vezes por um dos autores (RVM), e utilizou-se a média da DCEC e o menor valor da ECC.

A análise estatística foi realizada pelo programa SPSS 8.0. Histogramas de freqüência e o teste de Kolmogorov-Smirnov foram utilizados para avaliar a distribuição dos dados numéricos para características paramétricas. Após a identificação da não normalidade da amostra, optou-se por utilizar o teste de Mann-Whitney para comparar médias de amostras independentes e não paramétricas, considerando-se $\mathrm{p}<0,05$ estatisticamente significativo.

\section{Resultados}

Foram avaliados 29 olhos de 27 pacientes com diagnóstico de glaucoma submetidos a TREC. Onze $(37,9 \%)$ olhos foram submetidos a TREC com MMC e $18(62,1 \%)$ a TREC sem MMC.

A tabela 1 mostra, tanto para os olhos submetidos a TREC com MMC quanto para aqueles que foram submetidos a TREC sem MMC, características gerais dos pacientes: sexo, idade e olho operado, bem como dados sobre o exame oftalmológico deste pacientes: diagnósti- cos, número de medicações hipotensoras utilizadas, Po pré-operatória e relação escavação/disco (E/D). É importante salientar que quando comparamos o diagnóstico de glaucoma crônico simples (GCS) entre os dois grupos observamos uma diferença estatisticamente significativa $(\mathrm{p}=0,023)$.

A tabela 2 mostra, comparativamente entre os grupos com e sem MMC, os valores da ECC no préoperatório e com três e seis meses de pós-operatório, e a tabela 3 também compara entre o dois grupos as variações (subtração) da ECC com três e seis meses de pós-operatório. Verificou-se ausência de significância estatística quando confrontamos os valores dos dois grupos.

A tabela 4 mostra, comparativamente entre os grupos com e sem MMC, os valores da DCEC no préoperatório e com três e seis meses de pós-operatório, e a tabela 5 também compara entre o dois grupos as variações (subtração) da DCEC com três e seis meses de pósoperatório. Também, verificou-se ausência de significância estatística quando confrontamos os valores dos dois grupos.

A tabela 6 mostra, comparativamente entre os grupos com e sem MMC, os valores da área celular média no pré-operatório e com três e seis meses de pósoperatório, e a tabela 7 também compara entre o dois grupos as variações (subtração) da área celular média com três e seis meses de pós-operatório. Verificou-se, outra vez, ausência de significância estatística quando confrontamos os valores dos dois grupos.

Tabela 1

Características dos grupos submetidos a TREC com e sem mitomicina

\begin{tabular}{|c|c|c|}
\hline & $\begin{array}{c}\text { Com Mitomicina C } \\
n=11\end{array}$ & $\begin{array}{c}\text { Sem Mitomicina C } \\
n=18\end{array}$ \\
\hline Sexo & $\mathrm{M}-9(81,8 \%) / \mathrm{F}-2(18,2 \%)$ & M - $11(61,1 \%) / F-7(38,9 \%)$ \\
\hline Idade & $60,9 \pm 16,9$ anos & $63,3 \pm 15,1$ anos \\
\hline Olho & D - $8(72,7 \%) / E-3(27,3 \%)$ & $\mathrm{D}-6(33,3 \%) / \mathrm{E}-12(66,7 \%)$ \\
\hline \multirow[t]{2}{*}{ Diagnósticos } & GCS - $4(36,4 \%) /$ GPAF - $2(18,2 \%)$ & GCS $-15(83,3 \%) / \mathrm{GT}-1(5,5 \%)$ \\
\hline & GT - $2(18,2 \%) /$ GS - $2(18,2 \%) /$ GNV -1 $(9,0 \%)$ & / GPAF - $2(11,2 \%)$ \\
\hline \multicolumn{3}{|l|}{ Número de Medicações } \\
\hline \multirow[t]{4}{*}{ Pré-operatórias } & Três - $7(63,6 \%)$ & Duas - $9(50,0 \%)$ \\
\hline & Duas - $3(27,3 \%)$ & Três - $5(27,8 \%)$ \\
\hline & Uma - $1(9,1 \%)$ & Uma - $2(11,1 \%)$ \\
\hline & & Quatro - $2(11,1 \%)$ \\
\hline Po & $35,5 \pm 11,5 \mathrm{mmHg}$ & $24,0 \pm 6,9 \mathrm{mmHg}$ \\
\hline Relação ${ }^{E} / D$ & $0,7 \pm 0,2$ & $0,8 \pm 0,1$ \\
\hline
\end{tabular}

n: número de pacientes; M: masculino; F: feminino; D: direito; E: esquerdo;

GCS: glaucoma crônico simples, GPAF: glaucoma primário de ângulo fechado; GT: glaucoma traumático; GNV: glaucoma neovascular; GS: glaucoma secundário a facectomia. Po: pressão intra-ocular; $\mathrm{E} /$ : relação escavação/disco. 
Tabela 2

Espessura corneana central (média \pm desvio-padrão) pré-operatória e três e seis meses após a trabeculectomia

\begin{tabular}{cccc}
\hline $\begin{array}{c}\text { Espessura Corneal } \\
\text { Central }(\boldsymbol{\mu m})\end{array}$ & $\begin{array}{c}\text { Com Mitomicina } \\
\mathbf{C n}=\mathbf{1 1}\end{array}$ & $\begin{array}{c}\text { Sem Mitomicina C } \\
\mathbf{n}=\mathbf{1 8}\end{array}$ & $\begin{array}{c}\text { Valor de } \boldsymbol{p} \\
\text { (intergrupos) }\end{array}$ \\
\hline Pré-operatória & $539,4 \pm 43,8$ & $517,8 \pm 32,9$ & $0,270^{*}$ \\
3 meses & $527,5 \pm 41,9$ & $503,9 \pm 27,7$ & $0,120^{*}$ \\
6 meses & $531,9 \pm 38,4$ & $507,6 \pm 25,3$ & $0,072^{*}$ \\
\hline
\end{tabular}

n: número de pacientes; *Teste de Mann-Whitney.

Tabela 3

Médias e desvios-padrões das variações (subtração) da espessura corneana central três e seis meses após a trabeculectomia

\begin{tabular}{cccc}
\hline $\begin{array}{l}\text { Variação da Espessura } \\
\text { Corneal Central }(\boldsymbol{\mu m})\end{array}$ & $\begin{array}{c}\text { Com Mitomicina C } \\
\mathbf{n = 1 1}\end{array}$ & $\begin{array}{c}\text { Sem Mitomicina C } \\
\mathbf{n}=\mathbf{1 8}\end{array}$ & $\begin{array}{c}\text { Valor de } \boldsymbol{p} \\
\text { (intergrupos) }\end{array}$ \\
\hline 3 meses & $-11,9 \pm 25,7$ & $-13,9 \pm 16,7$ & $0,702^{*}$ \\
6 meses & $-7,5 \pm 28,1$ & $-10,2 \pm 15,6$ & $0,928^{*}$ \\
\hline
\end{tabular}

n: número de pacientes; *Teste de Mann-Whitney.

Tabela 4

Densidade das células endoteliais corneanas (média \pm desvio-padrão) pré-operatória e três e seis meses após a trabeculectomia

\begin{tabular}{cccc}
\hline $\begin{array}{c}\text { Densidade de Células } \\
\text { Endoteliais Corneais } \\
\text { (células } / \mathbf{~ m m}^{\mathbf{2}} \text { ) }\end{array}$ & $\begin{array}{c}\text { Com Mitomicina C } \\
\mathbf{n = 1 1}\end{array}$ & $\begin{array}{c}\text { Sem Mitomicina C } \\
\mathbf{n = 1 8}\end{array}$ & $\begin{array}{c}\text { Valor de } \boldsymbol{p} \\
\text { (intergrupos) }\end{array}$ \\
\hline Pré-operatória & $2.100,6 \pm 440,4$ & $2.328,9 \pm 451,6$ & $0,261^{*}$ \\
3 meses & $1.931,2 \pm 347,3$ & $2.128,2 \pm 399,2$ & $0,252^{*}$ \\
6 meses & $1.880,4 \pm 316,6$ & $2.051,4 \pm 376,5$ & $0,216^{*}$ \\
\hline
\end{tabular}

n: número de pacientes; *Teste de Mann-Whitney.

Tabela 5

Variações (subtração) da densidade de células endoteliais corneanas (média \pm desvio-padrão) três e seis meses após a trabeculectomia

\begin{tabular}{lccc}
\hline $\begin{array}{l}\text { Variação da Densidade } \\
\text { de Células Endoteliais } \\
\text { Corneais (células / } \mathbf{~ m m}^{2} \text { ) }\end{array}$ & $\begin{array}{c}\text { Com Mitomicina C } \\
\mathbf{n = 1 1}\end{array}$ & $\begin{array}{c}\text { Sem Mitomicina } \\
\mathbf{n = 1 8}\end{array}$ & $\begin{array}{c}\text { Valor de } \boldsymbol{p} \\
\text { (intergrupos) }\end{array}$ \\
\hline 3 meses & $-169,5 \pm 145,3$ & $-200,8 \pm 155,4$ & $0,486^{*}$ \\
6 meses & $-220,3 \pm 200,2$ & $-277,6 \pm 195,7$ & $0,393^{*}$ \\
\hline
\end{tabular}

n: número de pacientes; *Teste de Mann-Whitney. 
Tabela 6

Área celular média (média \pm desvio-padrão) pré-operatória e três e seis meses após a trabeculectomia

\begin{tabular}{cccc}
\hline $\begin{array}{c}\text { Área Celular } \\
\text { Média }\left(\boldsymbol{\mu m}^{\mathbf{2}}\right)\end{array}$ & $\begin{array}{c}\text { Com Mitomicina } \mathbf{C} \\
\mathbf{n = 1 1}\end{array}$ & $\begin{array}{c}\text { Sem Mitomicina C } \\
\mathbf{n = 1 8}\end{array}$ & $\begin{array}{c}\text { Valor de } \boldsymbol{p} \\
\text { (intergrupos) }\end{array}$ \\
\hline Pré-operatória & $496,1 \pm 109,2$ & $450,1 \pm 72,4$ & $0,345^{*}$ \\
3 meses & $538,6 \pm 110,2$ & $484,7 \pm 77,3$ & $0,251^{*}$ \\
6 meses & $527,6 \pm 91,8$ & $484,4 \pm 76,7$ & $0,312^{*}$ \\
\hline
\end{tabular}

n: número de pacientes; *Teste de Mann-Whitney

Tabela 7

Variações (subtração) de área celular média (média \pm desvio-padrão) três e seis meses após a trabeculectomia

\begin{tabular}{cccc}
\hline $\begin{array}{c}\text { Variação da Área } \\
\text { Celular Média }\left(\boldsymbol{\mu m}^{2}\right)\end{array}$ & $\begin{array}{c}\text { Com Mitomicina C } \\
\mathbf{n}=\mathbf{1 1}\end{array}$ & $\begin{array}{c}\text { Sem Mitomicina C } \\
\mathbf{n = 1 8}\end{array}$ & $\begin{array}{r}\text { Valor de } \boldsymbol{p}^{*} \\
\text { (intergrupos) }\end{array}$ \\
\hline 3 meses & $42,5 \pm 33,4$ & $34,6 \pm 52,4$ & $0,515^{*}$ \\
6 meses & $31,5 \pm 65,3$ & $34,3 \pm 41,0$ & $0,964^{*}$ \\
\hline
\end{tabular}

n: número de pacientes; *Teste de Mann-Whitney.

\section{Discussão}

As indicações do uso da MMC em TREC no Serviço de Glaucoma do Hospital São Geraldo/Hospital das Clínicas da UFMG são: reoperações em TRECs e glaucomas secundários (uveíte, trauma, glaucoma neovascular, glaucomas pos-facectomia, pós-transplantes, pós-vitrectomia e pós-retinopexia); além disso, devemos considerar alguns aspectos na decisão de se utilizar a MMC, como: considerar o seu uso em pacientes melanodérmicos e evitar utilizá-la em jovens devido ao risco de hipotonia grave, bem como infecções tardias. Acrescenta-se que este antimitótico, teoricamente, apresenta efeitos deletérios às células endoteliais corneanas e outras estruturas intra-oculares, como o corpo ciliar. Desse modo, o seu uso deve ser cuidadoso e com indicação criteriosa.

Neste estudo, o primeiro trabalho da literatura nacional a abordar esse tema na cirurgia do glaucoma, observamos uma diferença estatisticamente significativa $(\mathrm{p}=0,023)$ no diagnóstico de GCS em ambos os grupos, sendo que o grupo com MMC possuía $36,4 \%$ de pacientes com GCS e o grupo sem MMC 83,3\% de GCS. Salienta-se que no grupo com MMC, havia outros tipos de glaucoma ausentes no outro grupo: glaucoma neovascular e glaucoma secundário.
No presente estudo, verificou-se que $90,9 \%$ dos olhos submetidos a TREC com MMC utilizavam duas ou três medicações hipotensoras oculares previamente à cirurgia, enquanto que o mesmo ocorreu em $77,8 \%$ dos olhos do outro grupo, além disso, a Po média pré-operatória do grupo com MMC foi mais elevada que no grupo sem MMC $(p=0,05)$, demonstrando uma tendência a maior gravidade e refratariedade dos glaucomas no grupo com MMC, entretanto, sem significância estatística.

O número de medicações hipotensoras oculares e o nível da Po pré-operatórios podem justificar o menor valor médio da DCEC no grupo com MMC.Alguns autores acreditam que a baixa DCEC em pacientes glaucomatosos pode estar relacionada com níveis mais elevados de Po e o uso de um número elevado de medicações hipotensoras oculares. ${ }^{(9)}$ Outros autores observaram que a baixa DCEC está relacionada com a duração da elevação da Po. ${ }^{(5)}$

Apesar da média da escavação do disco óptico no grupo sem MMC ter sido um pouco maior que no grupo com MMC, esta diferença não foi estatisticamente significativa, sendo justificada pela característica crônica da doença no primeiro grupo.

É notório que a TREC, mesmo sem a utilização da MMC, pode acarretar perda de células do endotélio corneano que pode variar de $0,2 \%$ a $14,9 \%$, principal- 
mente nos casos que evoluem com toque iridocorneano pós-operatoriamente. ${ }^{(6)}$ Alguns autores ${ }^{(2)}$ observaram, após 29 dias de pós-operatório em média, uma redução média de $-32 \pm 210$ células $/ \mathrm{mm}^{2}$ na DCEC centrais de pacientes sem toque iridocorneano e naqueles com toque uma redução de $-250 \pm 210$ céls $/ \mathrm{mm}^{2}$. No presente estudo, após um período de acompanhamento pós-operatório maior (três e seis meses) verificamos uma redução da DCEC de -200,8 \pm 155,4 e -277,6 6 195,7 células/ $\mathrm{mm}^{2}$ naqueles pacientes submetidos a TREC sem MMC. Alguns estudos ${ }^{(2,6)}$ relatam que a TREC sem MMC pode acarretar edema e descompensação corneanas, entretanto, não realizaram a paquimetria para quantificar tais alterações. No grupo de TREC sem MMC observamos uma discreta diminuição da ECC em três e seis meses de pós-operatório $(-13,9 \pm 16,7 \mu \mathrm{m}$ e $-10,2 \pm$ $15,6 \mu \mathrm{m}$, respectivamente). Além disso, nosso estudo ainda acrescentou que as reduções da ECC observadas nos grupos com e sem MMC, quando comparadas entre si, não foram estatisticamente significativas.

A DCEC central foi comparada entre os dois grupos:TREC com e sem MMC. Verificou-se que não houve diferença estatisticamente significativa entre os dois grupos quando se comparou a variação da DCEC (tabela 5) em três e seis meses. Tal resultado diverge do encontrado no estudo conduzido por alguns autores que observaram diferença estatisticamente significativa na redução da DCEC entre os seguintes grupos:TREC sem MMC,TREC com MMC $(0,2 \mathrm{mg} / \mathrm{ml})$ e TREC com MMC $(0,4 \mathrm{mg} / \mathrm{ml})$, sendo maior quanto maior foi a concentração da MMC; entretanto, devemos salientar que no estudo acima a esponja de celulose embebida com MMC foi colocada abaixo do flap escleral, o leito foi lavado com $10 \mathrm{ml}$ de ringer lactato, o equipamento utilizado foi o Alcon PRO microscópio endotelial corneano, além disso, o seguimento foi de apenas três meses. ${ }^{(10)}$

Pesquisadores compararam a DCEC entre pacientes submetidos a TREC com MMC e pacientes submetidos a TREC suplementada com 5-Fluorouracil nas primeiras três semanas pós-operatórias e verificaram que não houve diferença estatisticamente significativa na redução da DCEC entre os grupos após um ano de seguimento. ${ }^{(1)}$ De acordo com esse estudo ${ }^{(1)}$ não há indícios de que a MMC acarrete elevadas perdas de células endoteliais, sendo tal afirmativa confirmada no presente estudo.

Ressalta-se que no presente estudo analisou-se a área celular média pré-operatoriamente e com três e seis meses de pós-operatório, verificando-se um aumento do seu valor após a TREC o que é justificado pelo trauma cirúrgico. Entretanto, quando comparamos os dois grupos, TREC com e sem MMC, não observamos diferença estatisticamente significativa.

Novos estudos que avaliem o endotélio corneano após TREC e outros procedimentos que utilizam a MMC, como o agulhamento, utilizando amostras com maior número de pacientes, são necessários para ratificar os dados aqui encontrados e obtermos maior segurança na utilização desse antimitótico.

\section{ConClusões}

1.ATREC com MMC não acarretou maior redução estatisticamente significativa da DCEC centrais, quando comparada à TREC sem MMC.

2. Também não houve variação estatisticamente significativa na ECC e na área celular média nos dois grupos.

\section{AbStRACt}

Purpose: To assess comparatively the central corneal thickness (CCT), the central corneal endothelial cells density (CECD) and mean area (CECAA), of patients who underwent trabeculectomy (Trab) with or without mytomicyn C (MMC). Methods: Prospective study. Twenty-nine eyes were divided in two groups: Trab with or without MMC. Ultrasonic pachymetry and non-contact specular microscopy were performed pre-operatively and three and six months after Trab. The mean value of the $C E C D$ and the low value of the CCT were used. Results: In the group of Trab with $M M C$, the variations of the CCT, $C E C D$ and $C E C A A$ values were, respectively: $-11.9 \pm 25.7$ $\mu \mathrm{m},-169.5 \pm 145.3 \mathrm{cells} / \mathrm{mm}^{2}$ and $42.5 \pm 33.4 \mu^{2}$ (three months); - 7.5 $\pm 28.1 \mu \mathrm{m},-220.6 \pm 200.2 \mathrm{cells} / \mathrm{mm}^{2}$ and $31.5 \pm 65.3 \mu \mathrm{m}^{2}$ (six months). In the group of Trab without $M M C$, the variations of the CCT, CECD and CECAA values were, respectively: - $13.9 \pm 16.7 \mu \mathrm{m},-200.8 \pm 155.4$ cells $/ \mathrm{mm}^{2}$ and $34.6 \pm 52.4 \mu^{2}$ (three months); - $10.2 \pm 15.6$ $\mu \mathrm{m},-277.6 \pm 195.7 \mathrm{cells} / \mathrm{mm}^{2}$ and $34.3 \pm 41.0 \mathrm{\mu m}^{2}$ (six months). Conclusion: After six months of follow-up, there was no difference in the variations of CCT, CECD and CECAA when Trab with MMC was compared with Trab without MMC.

Keywords: Cornea; Endothelium; Cell count; Microscopy;Trabeculectomy; Mytomicyn

\section{ReFERÊNCIAS}

1. Dreyer EB, Chaturvedi N, Zurakowski D. Effect of mitomycin $\mathrm{C}$ and fluorouracil-supplemented trabeculectomies on the anterior segment. Arch Ophthalmol. 1995; 113(5):578-80. 
2. Fiore PM, Ritcher CU, Arzeno G, Arrigg CA, Shingleton BJ, Bellows AR, Hutchinson BT. The effect of anterior chamber depth on endothelial cell count after filtration surgery. Arch Ophthalmol. 1989; 107(11):1609-11.

3. Sihota R, Lakshmaiah NC, Titiyal JS, Dada T, Agarwal HC. Corneal endothelial status in the subtypes of primary angle closure glaucoma. Clin Experiment Ophthalmol. 2003; 31(6):492-5.

4. Zarnowsky T, Lekawa A, Dyduch A, Turek R, Zagórski Z. Corneal endothelial density in glaucoma patients. Klin Oczna. 2005; 107(7-9):448-51. Polish

5. Ollivier FJ, Brooks DE, Komaromy AM, Kallberg ME, Andrew SE, Sapp HL, et al. Corneal thickness and endothelial cell density measured by non-contact specular microscopy and pachymetry in Rhesus macaques (Macaca mulatta) with laserinduced ocular hypertension. Exp Eye Res. 2003; 76(6):671-7.

6. Smith DL, Skuta GL, Lindenmuth KA, Musch DC, Bergstrom TJ. The effect of glaucoma filtering surgery on corneal endothelial cell density. Ophthalmic Surg. 1991; 22(5):251-5.

7. Fukuchi T, Hayakawa Y, Hara H, Abe H. Corneal endothelial damage after trabeculectomy with mitomycin $\mathrm{C}$ in two patients with glaucoma with cornea guttata. Cornea. 2002; 21(3):300-4.
8. Lázaro García C, Castillo Gómez A, García Feijóo J, Macía Benítez JM, García Sánchez J. Study of the cornea endothelium after glaucoma surgery. Arch Soc Esp Oftalmol. 2000; 75(2):75-80. Comment in: Arch Soc Esp Oftalmol. 2000; 75(10):657-8. Spanish.

9. Gagnon MM, Boisjoly HM, Brunette I, Charest M, Amyot M. Corneal endothelial cell density in glaucoma. Cornea. 1997; 16(3):314-8.

10. Sihota R, Sharma T, Agarwal HC. Intraoperative mitomycin C and the corneal endothelium. Acta Ophthalmol Scand. 1998; 76(1):80-2.

ENDEREÇO PARA CORRESPONDÊNCIA

Rafael Vidal Mérula

Rua Espírito Santo, 1315/402. Bairro Centro.

Juiz de Fora - MG.

CEP 36015-430

Fax: 3232168389

e-mail: rafaelmerula@hotmail.com 